\title{
Microsurgically Critical Anomaly of the Anterior Communicating Artery Complex During the Pterional Approach to a Ruptured Aneurysm: Double Fenestration of the Proximal $A_{2}$ Segments
} -Case Report-

\author{
Jun NAMIKI and Youichi DOUMOTO
}

Department of Neurosurgery, Ise-Keio Hospital, Keio University, Ise, Mie

\begin{abstract}
A 50-year-old man presented with a ruptured aneurysm of the anterior communicating artery (ACoA) complex anomaly with the neck of the aneurysm located in the upper ACoA. The right pterional approach was performed 2 days after admission. Retraction of the frontal lobe exposed the lower half of the ACoA complex consisting of the origins of the bilateral $A_{2}$ segments, and the apparent neck of the aneurysm was clipped. However, further exposure of the bilateral $A_{2}$ segments revealed double fenestration of the proximal $A_{2}$ segments, a subtype of duplication of the ACoA accompanied by a bridging artery between the upper and lower ACoA. The limited visualization of this ACoA complex anomaly with an aneurysm had led to the proximal origin of the bridging artery being mistaken for the neck of the aneurysm. Such double fenestration of the proximal $A_{2}$ segments is a microsurgically critical anomaly of the ACoA complex, because the bridging artery mimics the neck of an aneurysm when visualized by the pterional approach.
\end{abstract}

Key words: aneurysm, anterior communicating artery, duplication, fenestration, pterional approach

\section{Introduction}

Fenestration or duplication of the intracranial arteries associated with saccular aneurysms are not rare, and commonly affect the anterior communicating artery (ACoA). ${ }^{7)}$ Fenestration of the ACoA is usually described as duplication of the ACoA, because these malformations are sometimes difficult to differentiate. Double fenestration of the proximal $A_{2}$ segments is a subtype of duplication of the ACoA, and consists of duplication of the ACoA accompanied by a bridging artery between the upper and lower ACoA which creates double fenestration in the proximal $A_{2}$ segments (Fig. 1). However, no special attention has been paid to this subtype of the ACoA complex anomaly, which may lead to confusion and misidentification of the aneurysm neck during microsurgery through the pterional ap- proach. The pterional approach is commonly used to treat aneurysms of the anterior circulation in the basal cerebral arterial circle of Willis, except for ACoA aneurysms with necks over $2 \mathrm{~cm}$ above the anterior clinoid process. ${ }^{8)}$

We describe a case of ruptured aneurysm associated with this ACoA complex anomaly in which the neck of the aneurysm was located in the upper $\mathrm{ACoA}$, and the proximal origin of the bridging artery was mistaken for the neck of the aneurysm during microsurgery through the pterional approach, because the lower half of the ACoA complex came into view first and mimicked the visualization of the five vessels composing the bilateral $A_{1}$ segments, the origins of the bilateral $A_{2}$ segments, and the ACoA, which must be identified before clipping ACoA aneurysms.

Received October 7, 2002; Accepted February 3, 2003

Author's present address: J. Namiki, M.D., Department of Emergency and Critical Care Medicine, School of Medicine, Keio University, Tokyo, Japan. 


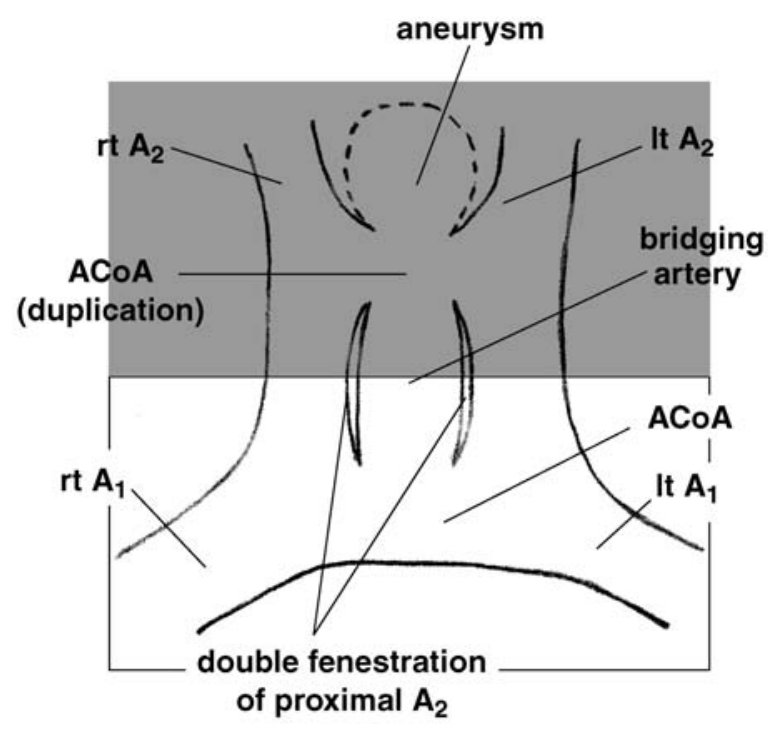

Fig. 1 Schematic drawing showing double fenestration of the proximal $A_{2}$ segments. This anterior communicating artery (ACoA) complex anomaly is classified as duplication of the ACoA accompanied by a bridging artery between the upper and lower ACoA. In the present case, a ruptured aneurysm projected upwards with the neck located in the upper ACoA. The bridging artery created a double fenestration in the proximal $A_{2}$ segments. Retraction of the frontal lobe by the pterional approach first revealed the lower part of the double fenestration (clear area), whereas the upper part of the double fenestration with the aneurysm was not visible (shaded area). This limited view suggests that the proximal origin of the bridging artery is the neck of the aneurysm.

\section{Case Report}

A 50-year-old man suddenly lost consciousness while watching television at home on October 9, 2000 and was brought to our hospital by ambulance. $\mathrm{He}$ recovered consciousness about 10 minutes later, but complained of severe headache and mild weakness and numbness in his right lower extremity. Head computed tomography (CT) showed subarachnoid hemorrhage (Fig. 2A). Cerebral angiography revealed a saccular aneurysm arising from the junction between the bilateral $\mathrm{A}_{2}$ segments and ACoA, with the neck $10 \mathrm{~mm}$ above the anterior clinoid process (Fig. 2B, C). No malformation of the ACoA complex was found.

The right pterional approach was performed on October 11, 2000. His neurological condition was World Federation of Neurological Surgeons grade II immediately before surgery. After reaching the ACoA complex under the operating microscope, temporary clips were applied to the left and right $A_{1}$ segments, and the origins of the bilateral $A_{2}$ segments and the apparent neck of the aneurysm between them were identified (Fig. 3A). The first clipping was completed without further visualization requiring dissection in the vicinity of the aneurysm dome (Fig. 3B). After distally exposing the bilateral $A_{2}$ segments from their origins by removing the hematoma in the interhemispheric fissure, another ACoA was discovered. The vessel clipped as the aneurysm neck arose from the lower ACoA and ended at the upper ACoA, forming double fenestration of the proximal $A_{2}$ segments (Fig. 3C). This bridging artery between the lower and upper ACoA appeared to be the neck of an aneurysm when the
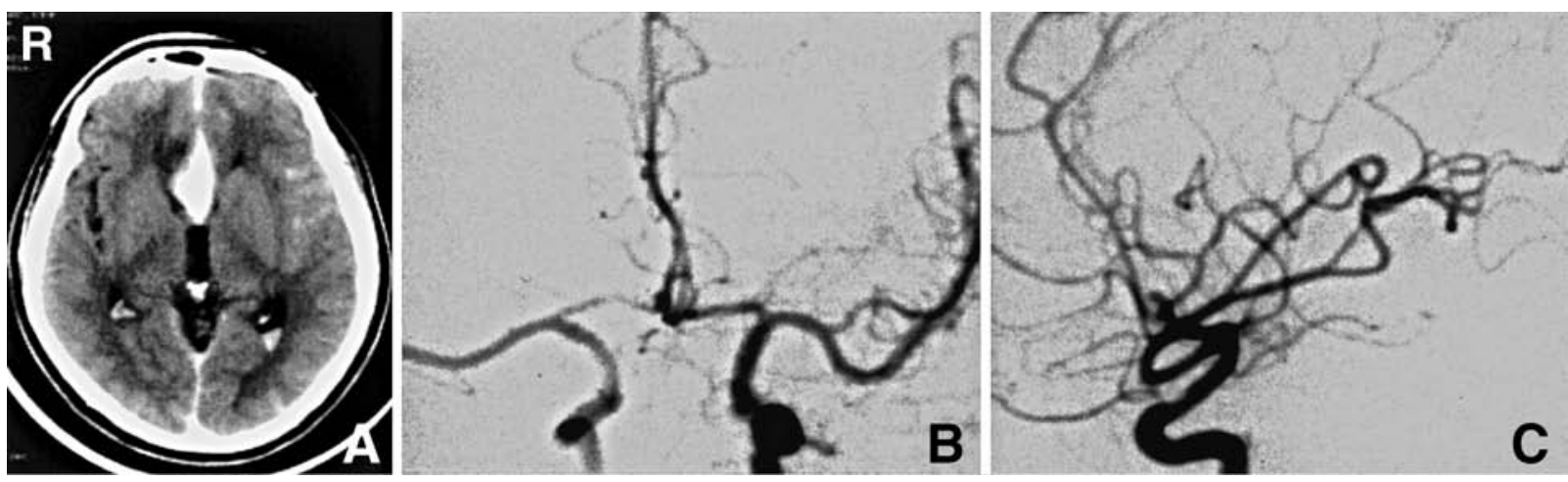

Fig. 2 A: Head computed tomography scan before surgery showing subarachnoid hemorrhage, especially in the interhemispheric fissure, suggesting rupture of an anterior communicating artery (ACoA) aneurysm. B, C: Left carotid angiograms before surgery showing an ACoA aneurysm between the bilateral $A_{2}$ segments on the anteroposterior view (B) and the lateral view (C). No anomaly of the ACoA complex can be detected. 

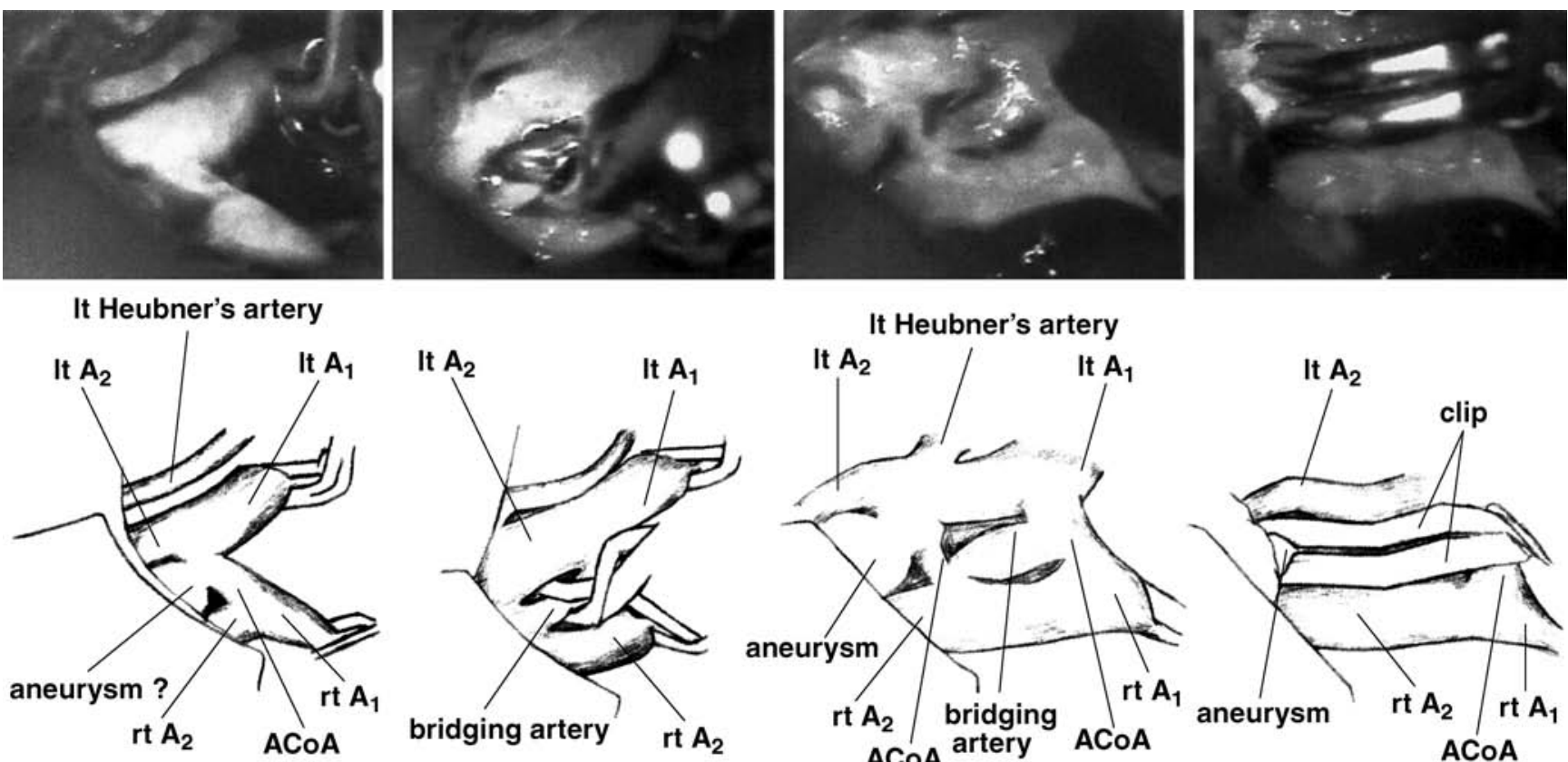

A
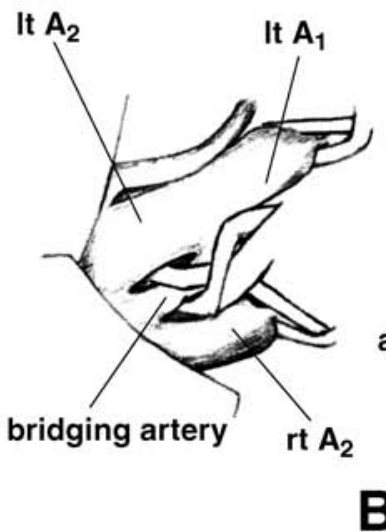

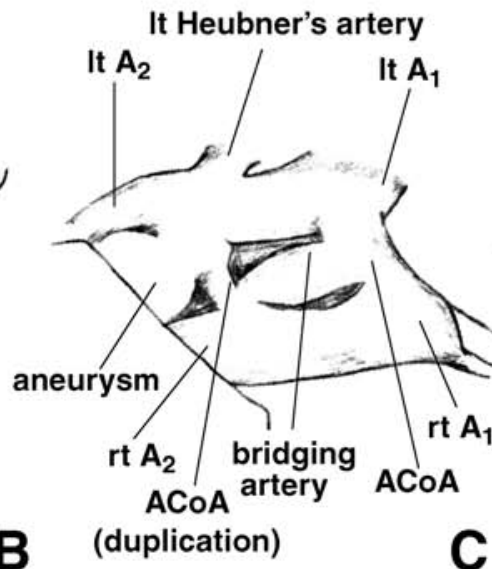

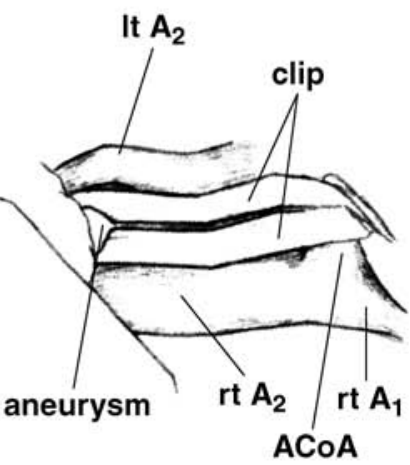

D

Fig. 3 Video still images captured during the microsurgery procedure through the right pterional approach (upper row) and corresponding schemas (lower row). A: The bilateral $A_{1}$ segments (temporarily clipped), the anterior communicating artery (ACoA), and the lower part of the double fenestration composed of the proximal segments of the bilateral $A_{2}$ and the bridging artery mimicking an aneurysm are visualized. B: The double fenestration was dissected, and the bridging artery was clipped as the neck of the aneurysm. C: Further visualization of the ACoA complex revealed that the bridging artery mimicked the neck of the aneurysm and the true neck of the aneurysm was located on the upper ACoA. D: The true neck of the aneurysm was clipped.

lower half of the ACoA complex was viewed through the pterional approach. The true neck of the aneurysm was subsequently identified on the upper ACoA and clipped (Fig. 3D).

Although the patient suffered symptomatic vasospasm and disorientation for a few days after surgery, he was discharged from the hospital 4 weeks after admission without neurological deficits or signs of cerebral infarction on CT. Postoperative angiography confirmed the disappearance of the aneurysm but did not reveal the fenestration of the ACoA complex. Retrospective examination of the first cerebral angiography could not detect the vascular malformation.

\section{Discussion}

The pterional approach is widely used for microsurgery of aneurysms of the anterior circulation in the basal cerebral arterial circle of Willis and some aneurysms of the posterior circulation, including those located at the tip of the basilar artery. ACoA aneurysms are mainly treated by the pterional and the interhemispheric approaches. The interhemispheric approach is more appropriate for aneurysms with necks arising from the ACoA and more than $2 \mathrm{~cm}$ from the anterior clinoid process, ${ }^{8)}$ because visualization of the ACoA complex by the pterional approach in such cases generally requires extreme retraction of the frontal lobe or considerable resection of the gyrus rectus. Most other ACoA aneurysms can be treated by the pterional approach. Since only a limited number of aneurysm cases are referred to neurosurgeons in Japan, and there are no specialists who operate on large numbers of such cases, the pterional approach is important to master, because it is widely adaptable to most aneurysm cases.

Five vessels of the ACoA complex must be identified before dissection of the aneurysm neck: the bilateral $A_{1}$ and $A_{2}$ segments, and the ACoA. ${ }^{8)}$ The integrity of the hypothalamic artery emerging from the ACoA should also be assured, especially if the aneurysm projects backward (the dorsal posterior 
type in the classification of ACoA aneurysms ${ }^{2}$ ). In the pterional approach, the neck of an ACoA aneurysm projecting upward is seen between the origins of the bilateral $A_{2}$ segments from the ACoA, as in our patient, so an anomalous artery arising from the ACoA and passing between the bilateral $A_{2}$ segments can be mistaken for the aneurysm neck unless the aneurysm is fully exposed.

One of the most commonly encountered of such anomalous arteries is the median artery of the triplicate anterior cerebral artery (ACA), called the median artery of the corpus callosum (MACC) or the accessory ACA.4) Double fenestration of the proximal $\mathrm{A}_{2}$ segments is another such anomaly of the ACoA complex in which a vessel emerges from the junction between the ACoA and the bilateral $A_{2}$ segments, and is a subtype of duplication of the ACoA accompanied by a bridging artery. However, this microsurgically critical anomaly has never been described with special reference to clipping of a ruptured aneurysm on the ACoA.

The incidence of double fenestration of the proximal $A_{2}$ segments is unknown, whereas the incidence of duplication of the ACoA is as high as $5.7 \%$ to $11.1 \%$ in patients with an ACoA complex aneurysm..$^{1,3,5,6)}$ Some cases of aneurysm with double fenestration of the proximal $\mathrm{A}_{2}$ segments were classified as duplication of the ACoA. ${ }^{5,9)}$ Therefore, we think that double fenestration of the proximal $A_{2}$ segments is not particularly infrequent.

The MACC or the accessory ACA can be detected preoperatively by careful angiographic evaluation, ${ }^{5)}$ whereas duplication of the ACoA is seldom identified preoperatively even by detailed angiography. ${ }^{6}$ ) Therefore, we emphasize that duplication of the ACoA with a bridging artery, or double fenestration of the proximal $A_{2}$ segments, is usually occult on preoperative angiography, but is critical to consider in cases with an ACoA aneurysm projecting upward, because the bridging artery can be mistaken for the neck of an aneurysm if the ACoA aneurysm is treated by the pterional approach. After clipping the neck of an ACoA aneurysm projecting upward, complete exposure of the aneurysm is recommended to confirm the anatomical orientation and avoid misclipping of an anomalous vessel present in this type of ACoA complex anomaly.

The domes of ACoA aneurysms projecting upward are sometimes buried in the brain and are difficult to explore without extensive damage to the frontal cortex. Alternatively, the bilateral $\mathrm{A}_{2}$ seg- ments can be exposed distally from the junction with the ACoA, where the aneurysm neck is located, by removing the hematoma in the interhemispheric fissure after clipping the neck of the aneurysm. Exposure of the bilateral $A_{2}$ segments also helps to differentiate the neck of the ACoA aneurysm from any anomalous vessel that emerges from the junction between the ACoA and the bilateral $A_{2}$ segments, except the MACC and the accessory ACA, which can be detected by preoperative angiography.

\section{References}

1) Inoue T, Matsushima T, Fujii K, Suzuki S, Fukui M, Hasuo K, Matsuno H: Microsurgical and angiographic analysis of anterior communicating artery aneurysms with associated anomalies. Fukuoka Igaku Zasshi 83: 397-402, 1992

2) Kobayashi S, Ichinose Y, Sugita K: [Surgery of the anterior communicating artery aneurysm. Anatomical considerations]. No Shinkei Geka 15: 235-240, 1987 (Jpn)

3) Kwak R, Niizuma H, Hatanaka M, Suzuki J: Anterior communicating artery aneurysms with associated anomalies. J Neurosurg 52: 162-164, 1980

4) Morioka M, Fujioka S, Itoyama Y, Ushio Y: Ruptured distal accessory anterior cerebral artery aneurysm: case report. Neurosurgery 40: 399-401, 1997

5) Nathal E, Yasui N, Sampei T, Suzuki A: Intraoperative anatomical studies in patients with aneurysms of the anterior communicating artery complex. J Neurosurg 76: 629-634, 1992

6) Ogawa A, Suzuki M, Sakurai Y, Yoshimoto T: Vascular anomalies associated with aneurysms of the anterior communicating artery: microsurgical observations. J Neurosurg 72: 706-709, 1990

7) San-Galli F, Leman C, Kien P, Khazaal J, Phillips SD, Guerin J: Cerebral arterial fenestrations associated with intracranial saccular aneurysms. Neurosurgery 30: 279-283, 1992

8) Sano H: [Surgical approach for aneurysm of the anterior communicating artery]. No Shinkei Geka 28: 9-16, 2000 (Jpn)

9) Yasargil MG: Microneurosurgery I. Stuttgart, Georg Thieme Verlag, 1984, pp 92-128

Address reprint requests to: J. Namiki, M.D., Department of Emergency and Critical Care Medicine, School of Medicine, Keio University, 35 Shinano-machi, Shinjuku-ku, Tokyo 160-8582, Japan.

e-mail: namiki@sc.itc.keio.ac.jp. 